\title{
Phytochemical map leads to local omics world
}

\author{
Ryo Nakabayashi \\ RIKEN Center for Sustainable Resource Science, 1-7-22 Suehiro-cho, Tsurumi-ku, Yokohama 230-0045, Japan \\ Author for correspondence: R. Nakabayashi, ryo.nakabayashi@riken.jp
}

\begin{abstract}
Summary: Imaging mass spectrometry (IMS) is an analytical method for visualizing the localization of metabolites. IMS analysis involves performing spatial mass spectrometry in coordinates specified on cross-section or longitudinal section of organisms, including plants. The localization of detected metabolites can be visualized using the $\mathrm{m} / \mathrm{z}$ value and signal intensity acquired in IMS analysis. The development of genome or transcriptome sequencing technologies enables us to perform extremely local part analyses at the level of the cells, tissues, or organs. Using the sequencing technologies, phytochemical genomics researches have shown that specialized metabolic pathways have associations between gene expression and metabolite accumulation in a tissueor organ-specific manner. These findings suggest that the identification of specialized metabolites in the local parts through IMS analysis makes narrowing down biosynthetic genes more simplified. The IMS analysis is potentially capable of increasing the efficiency and accuracy in phytochemical genomics. Herein, I provide recent updates on IMS analysis in plants.
\end{abstract}

Key words: imaging mass spectrometry, metabolomics, phytochemical genomics, specialized metabolite

\section{INTRODUCTION}

Mass spectrometry (MS) is one of the most frequently used methods for the analysis of metabolites in metabolomics (Tsugawa 2018, Wolfender et al. 2019). MS analysis enables us to acquire structural information, such as $\mathrm{m} / \mathrm{z}$ value, signal intensity, and isotopic pattern of precursor ions derived from metabolites with high sensitivity and coverage. In tandem MS (MS/MS) analysis, more structural information, including substructures, can be acquired from product ions and neutral losses, which are fragments of the precursor ions. For reducing the effect of ion suppression and acquiring better quality data from as many metabolites as possible, another method is used for separating the metabolites before MS or MS/MS analysis. The selected method varies according to the physicochemical properties of target metabolites; for instance, gas chromatography is used for volatile and derivatized primary metabolites, capillary electrophoresis is used for ionic metabolites, and liquid chromatography (LC) is used for lipids and specialized metabolites (Saito and Matsuda 2010, Lei et al. 2011). In LC-MS or LC-MS/MS analysis, retention time can be used for estimating the physicochemical properties as well as the above-described data in MS or MS/MS analysis. Polyhedral data of a metabolite is useful for its chemical assignment.

Imaging mass spectrometry (IMS) is an analytical method for visualizing the localization of metabolites by adding spatial data to MS data in cross or longitudinal section of organisms (Buchberger et al. 2018). Several types of MS can be used for IMS analysis; these include secondary ion, desorption electrospray ionization, laser ablation electrospray ionization, and matrix-assisted laser desorption ionization (MALDI) (Lee et al. 2012, Addie et al. 2015, Etalo et al. 2015). Of them MALDI is widely used in IMS analysis (Fujimura and Miura 2014, Li et al. 2014, Yamamoto et al. 2016, Baker et al. 2017, Duenas et al. 2017, Kompauer et al. 2017, 2017, Shariatgorji et al. 2019, Yamamoto et al. 2019). Along with the section of the organisms, MALDI-IMS analysis requires a matrix reagent for helping to ionize metabolites on the surface of the section. Spraying the matrix reagent dissolved in an organic solvent onto the section and drying the solvent multiple times helps to extract metabolites on the surface and crystallize the matrix with the metabolites. MS analysis according to the coordinates in the section provides spatial data. Spatial MS analyzes the matrix-sprayed section via laser pulse. The range of laser pulses can be set in an arbitrary manner. The localization of detected metabolites is visualized using the signal intensities of ions on the coordinates (Figure 1).

Phytochemical genomics is the study of the identification of genes involved in biosynthetic pathways of primary metabolites and specialized metabolites in plants (Saito 2013). Thus far, many of the biosynthetic genes have been identified in the pathways of specialized metabolites (Fernie and Tohge 2017, Alseekh and Fernie 2018). Multi-omics of genomics/transcriptomics and metabolomics analyses have shown associations between expression of genes and accumulation of metabolites in a tissueor organ-specific manner (Yonekura-Sakakibara et al. 2008, Suzuki et al. 2015, Li et al. 2016, Tohge et al. 2016, Colinas and Goossens 2018). The manner suggests that "cobehavior" analysis using transcriptome and metabolome data in samples more accumulating arbitrary metabolites and less makes narrowing down candidate genes easier (Rai et al. 2017). Recently, genome or transcriptome sequencing technologies have achieved remarkable breakthroughs. These technologies provide us with a chance to perform multi-omics analysis at the level of the cells, tissues, or organs. It is important to obtain data from only target parts, but not from extraneous parts in the spacial analyses. Reducing contamination increase the quality of data in the target parts (Figure 2).

It is expected that combining IMS analysis with other -omics study increases the efficiency and accuracy of narrowing down candidate genes in phytochemical genomics in a local manner by identifying the localization of target metabolites. Due to the mechanical issues such as the limitation of spatial resolution by laser, performing MALDI-IMS analysis is challenging at the 
single-cell or organelle level. However, recent approaches have shown that this method can be used for metabolome analysis at the tissue- or organ-level. Herein, I reviewed recent updates on the use of MALDI-IM analysis in plants. The general principles, experimental procedures, and examples of studies that utilize this technique have been well-described in previous reviews (Boughton et al. 2016, Dong et al. 2016). spraying matrix reagent

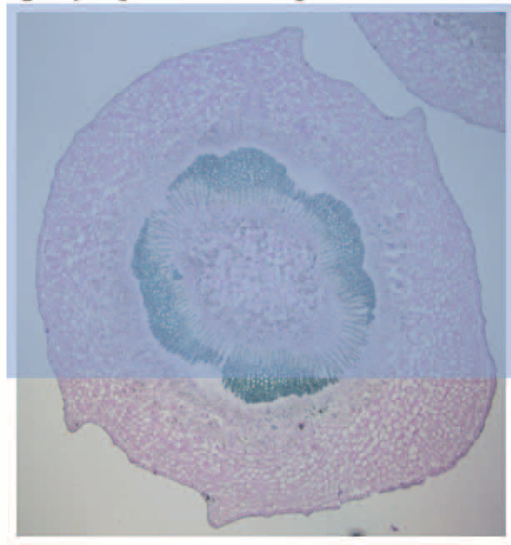

obtaining mixed crystal

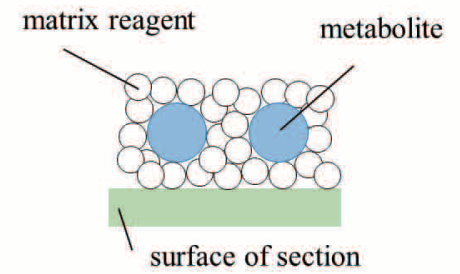

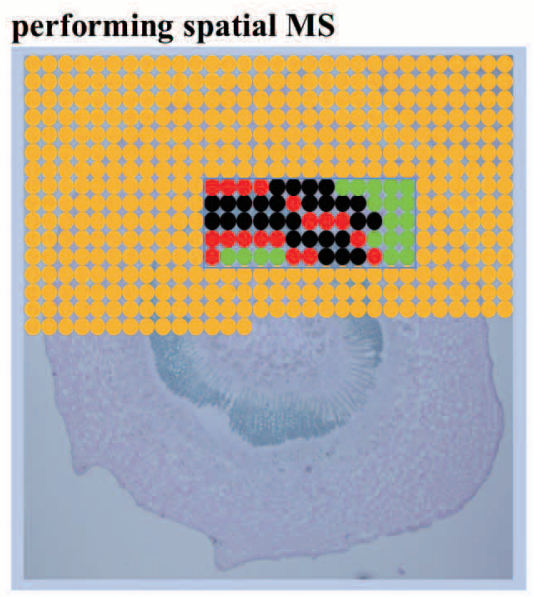

acquiring spatial MS data

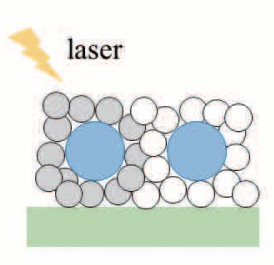

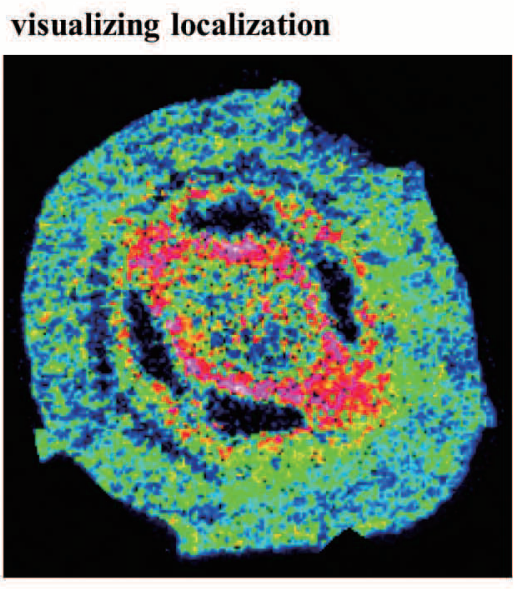

selecting arbitrary $m / z$ value
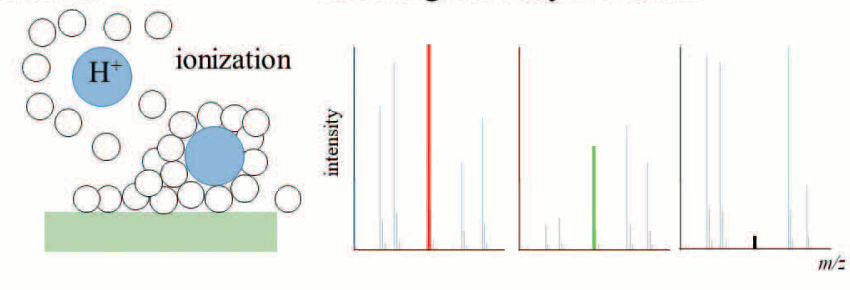

Figure 1. Overview of matrix-assisted laser desorption ionization-imaging mass spectrometry.

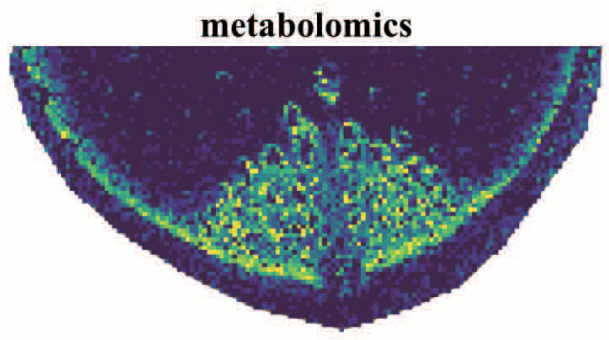

imaging mass spectrometry for characterizing local metabolite accumulation
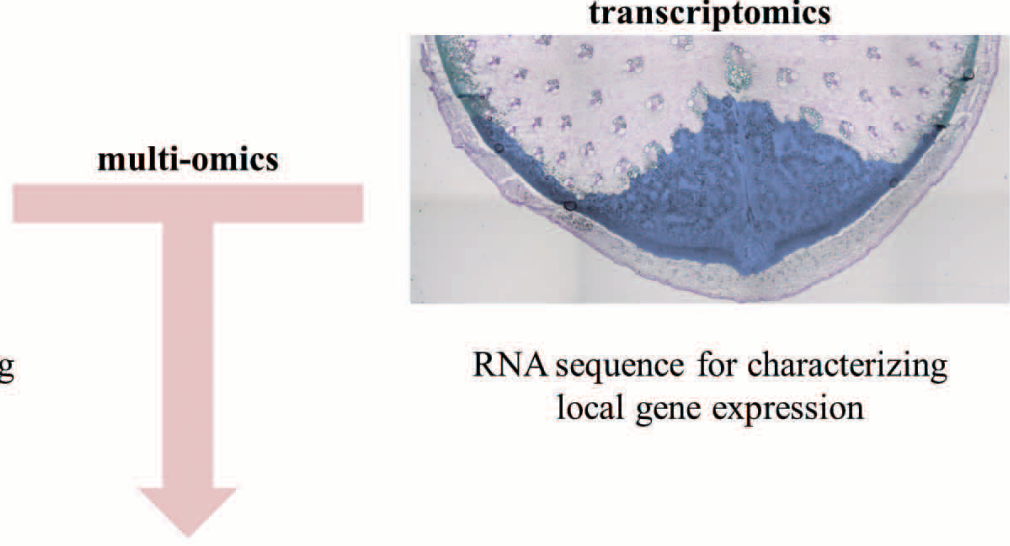

RNA sequence for characterizing local gene expression

identification of tissue/organ-specific metabolism

Figure 2. Strategy of multi-omics analysis using imaging mass spectrometry. 


\section{WORKFLOW OF MALDI-IMS ANALYSIS}

Generally, MALDI-IMS analysis consists of the following seven steps: 1) estimation of data size, 2) image of figure, 3) preparation of section, 4) selection of matrix reagent, 5) spraying the reagent, 6) data acquisition, and 7) data analysis. Understanding every single step is necessary to maximize reproducibility and to produce good quality data.

\section{ESTIMATION OF DATA SIZE}

Understanding the size of dataset is important for handling IMS platform. Previous studies using ultrahigh-resolution MALDI-IMS analysis provided raw data at the gigabyte scale during each run (Nakabayashi et al. 2017, 2019). Although it is expected that other instruments of IMS analysis may provide less data, a high-spec computational environment is required for data acquisition, storage, and processing of IMS data.

\section{IMAGE OF FIGURE}

Images of figures for publication need to be considered before the analysis. By considering other data, it can be decided which cross or longitudinal section is more appropriate. Since limited space is available on a glass slide, bigger leaves may often not fit on the slide. However, if the leaf is rolled, IMS analysis can be performed on its cross or longitudinal section. For instance, MALDI-IMS analysis was used for analyzing a cross-section of a rolled leaf from Catharanthus roseus, a monoterpene indole alkaloid (MIA)-producing medicinal plant (O'Connor and Maresh 2006). The IMS analysis showed the localization of MIAs in the leaf tissues (Nakabayashi et al. 2017).

Labeling the stable isotopes produces a mass shift of $\mathrm{m} / \mathrm{z}$ values between stable isotope-labeled and non-labeled metabolites in MS and MS/MS analysis (Giavalisco et al. 2011, Nakabayashi et al. 2013, Glaser et al. 2014, Wang and Jones 2014, Nakabayashi et al. 2015, Nakabayashi et al. 2016, Freund and Hegeman 2017, Kera et al. 2018, Hautbergue et al. 2019, Tsugawa et al. 2019). It is expected that stable isotope- and non-labeled metabolites can be mainly detected in stable isotopeand non-labeled plants, respectively. Stable isotope-labeled and non-labeled metabolites can not be detected in the non-labeled and stable isotope-labeled plants, respectively. Comparative analyses showed that the mirror image of stable isotope-labeled and non-labeled metabolites can not be obtained (Nakabayashi et al. 2017).

\section{PREPARATION OF SECTION}

Fresh plant samples are often required for IMS analysis. Therefore, for analyzing transformants, mutants, or harmful plants, the administration must allow the importation of these plants into the laboratory.

Fresh samples are frozen to quench enzymatic and metabolic activities, and their shapes are maintained using embedding reagent. Using a block of the reagent-embedded sample, one or a few sections are cut and placed on a glass slide and freeze-dried. The freeze-drying process often results in peeling or warpage due to the removal of water content from the section or another mechanical reason. Moreover, laser pulses may cause deformation of the section. Therefore, MALDI-IMS analysis requires that section withstands to thousands or tens of thousands of laser pulses. For that the section needs to tightly adhere to the slide. Recently, a technique called the NakaMi method has been developed for helping the section to adhere to the slide using transfer tape and conductive double-sided tape (Figure 3) (Nakabayashi et al. 2019). This method can be used for preparing sections of fruits, flowers, leaves, petioles, stems, and roots.
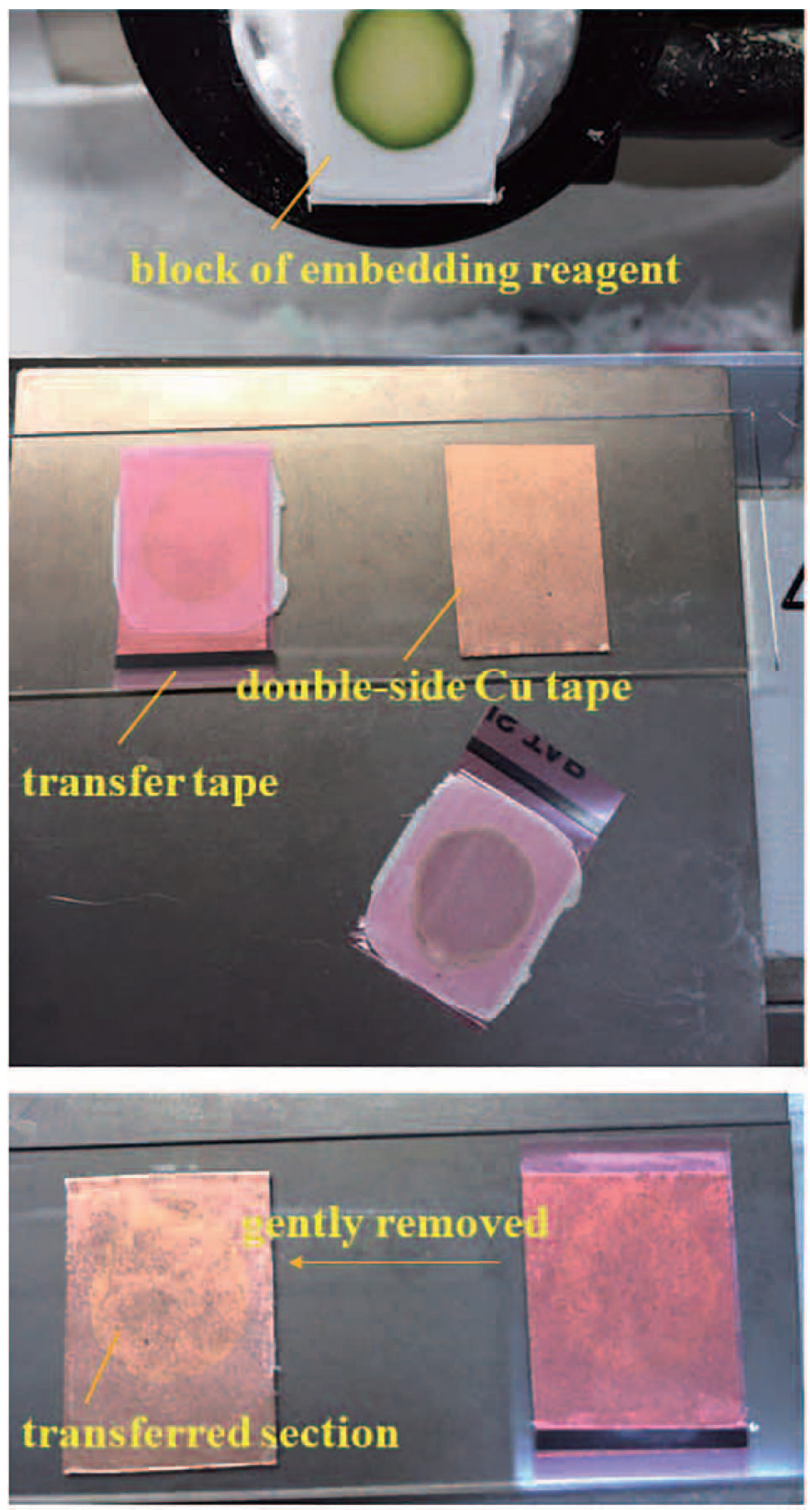

Figure 3. Overview of NakaMi method.

\section{SELECTION OF MATRIX REAGENT}

Several matrix reagents may be used for visualizing the localization of phytochemicals; primary metabolites (carbohydrates, lipids, and fatty acids); and specialized metabolites (alkaloids, glucosinolates, and flavonoids) (Boughton et al. 2016, Dong et al. 2016). For analyzing a 
single metabolite or a metabolite group, the reported reagents are sufficient. However, there is limited information available regarding which matrix reagent should be selected for analyzing a wide range of specialized metabolites. A previous study used $\alpha$-cyano-4-hydroxycinnamic acid (CHCA), 1,5-diaminonaphthalene (DAN), 2,5-dihydroxybenzoic acid (DHB), and 3-hydroxypicolinic acid (HPA) as matrix reagents to analyze 36 metabolites belonging to 12 groups (Nakabayashi et al. 2019). The metabolites were analyzed under the same analytical conditions (Table 1). The results suggested that both CHCA and DHB are appropriate for detecting a wide range of metabolites in positive ion mode. The coverage of the specialized metabolites indicated that MALDI-IMS analysis can be used for metabolomics.

\section{SPRAYING THE MATRIX REAGENT}

As described above, spraying the matrix reagent is essential for obtaining mixed crystals containing the matrix and extracted metabolites. Spraying the matrix reagent in excess results in ion suppression due to the high amount of the matrix. Meanwhile, spraying insufficient matrix reagent results in poor detection of metabolites due to the low quantity of extracted metabolites. A small crystal size results in high-quality spatial data. For obtaining good quality data, the matrix reagent must be uniformly sprayed onto the section multiple times to prepare fine crystals. Manual spraying may be performed using an airbrush, but this method requires a high skill level. Alternatively, an automatic sprayer may be used for creating uniform crystals. There are several commercial sprayers available; the appropriate sprayer must be selected on the basis of the advantages; it provides in terms of spray, extraction, time, and warming.

\section{DATA ACQUISITION}

Before initiating the analysis, the range of laser pulses should be set according to the signal intensity of ions derived from the metabolites. When metabolites are easily ionized and provide high signal intensity, the range of laser pulses can be set to small $(<50 \mu \mathrm{m})$. However, when metabolites are not easily ionized, the range must be set to large $(>100 \mu \mathrm{m})$. The time of analysis depends on the data points and the number of pulses. It may take several hours to acquire all the data.

\section{DATA ANALYSIS}

For understanding the localization of metabolites, tens of thousands of data points must be processed. Existing analytical approaches and software tools enable us to routinely identify the general or specific localization of metabolites, including specialized metabolites (Nakabayashi et al. 2019). Segmentation analysis using the vendor software SCiLS Lab (Bruker Daltonics, Germany) provides a top-down clustering using bisecting $k$-means that allows for the arbitrary segmentation of metabolite features in a reasonable time frame. The output figure is a map that reflects the localization of analyzed metabolites using color labeling. The localization of metabolites in the spear and root of asparagus (Asparagus officinalis) is identified using this method (Figure 4). This method is helpful for a quick understanding of tissue-redundant metabolites.

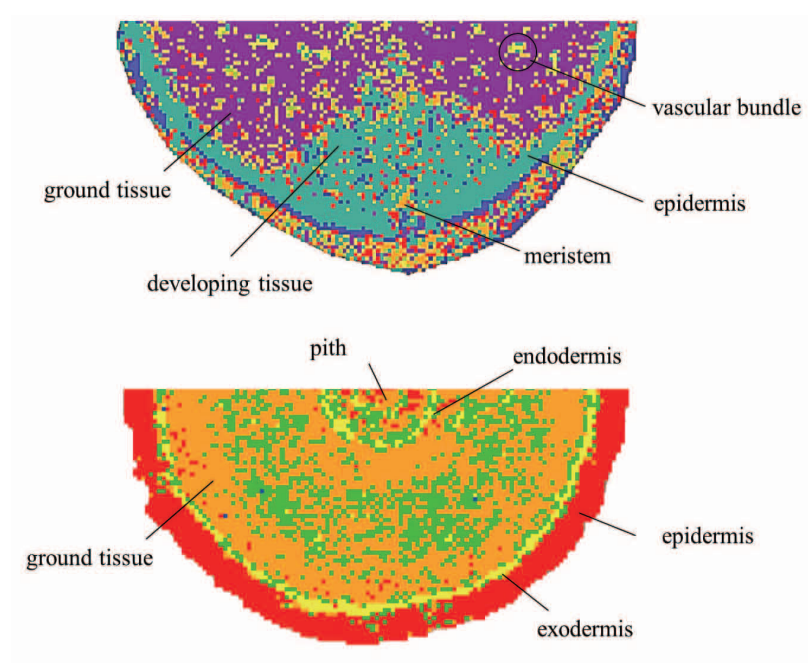

Figure 4. Segmentation analysis in asparagus (Asparagus officinalis). The bisecting $k$-means analysis provides patterns of the accumulation of detected metabolites. Each color indicates a pattern meaning that metabolites accumulate more at the part than the others.

Upper, spear; lower, root.

\section{FUTURE PERSPECTIVE}

MALDI-IMS is a non-separation analytical method that results in ion suppression because hundreds of metabolites are probably analyzed at a single data point. This is a limitation of acquiring MS/MS data in sections. For analyzing the metabolites at a higher level of sensitivity, this method needs to be combined with a separation technique. Introducing ion mobility (IM) to MS or MS/MS analysis enables us to separate metabolites, increase detected metabolites, and improve the quality of data. This method is also capable of separating isobaric structural isomers and analogs (Schroeder et al. 2019). Plants produce structural isomers and analogs. It is, therefore, expected that IM-MALDI-IMS analysis (Spraggins et al. 2019) provides new insights into the role of the structural isomers/analogs on specialized metabolites.

\section{CONCLUSION}

Here, I have summarized the recent updates on MALDI-IMS analysis and its potential capacity for enhancing researches in phytochemical genomics. For identifying the function of genes, multiomics analysis of transcriptomics and metabolomics analysis is performed. Comparative analysis using transformants/ mutants has allowed for the identification of the function of metabolites (Nakabayashi et al. 2014, Nakabayashi and Saito 2015, Massalha et al. 2017, Huang et al. 2019). As described at the beginning of this review, polyhedral data is important for understanding the metabolite. The addition of spatial data to MS data provides insights regarding the biological and physiological functions of metabolites. Plant specialized metabolites may help mitigate environmental stress such as biotic and abiotic 
Table 1. Screening of matrix regent using specialized metabolites.

\begin{tabular}{|c|c|c|c|c|c|c|c|c|}
\hline type & name & molecular formila & addact type & exact mass & CHCA & DAN & DHB & HPA \\
\hline \multirow{6}{*}{ alkaloid } & eserine & $\mathrm{C}_{15} \mathrm{H}_{21} \mathrm{~N}_{3} \mathrm{O}_{2}$ & {$[\mathrm{M}+\mathrm{H}]^{+}$} & 276.170653 & ++ & + & +++ & ++++ \\
\hline & & & {$[\mathrm{M}+\mathrm{Na}]^{+}$} & 298.152598 & +++ & N.D. & +r+t & ++ \\
\hline & kopsinine & $\mathrm{C}_{21} \mathrm{H}_{26} \mathrm{~N}_{2} \mathrm{O}_{2}$ & $\lceil\mathrm{M}+\mathrm{H}\rceil^{+}$ & 339.206705 & +++ & N.D. & ++++ & ++ \\
\hline & & & {$[\mathrm{M}+\mathrm{Na}]^{+}$} & 361.188649 & ++++ & N.D. & +++ & N.D. \\
\hline & voacamine & $\mathrm{C}_{43} \mathrm{H}_{52} \mathrm{~N}_{4} \mathrm{O}_{5}$ & {$[\mathrm{M}+\mathrm{H}]^{+}$} & 705.401047 & ++ & + & ++++ & N.D. \\
\hline & & & $\lceil\mathrm{M}+\mathrm{Na}\rceil^{+}$ & 727.382991 & ++ & +++ & N.D. & ++r+ \\
\hline \multirow{6}{*}{ anthocyanidin } & cyanidin & $\mathrm{C}_{15} \mathrm{H}_{11} \mathrm{O}_{6}$ & $\mathrm{M}^{+}$ & 287.055014 & +++ & ++ & +++++ & N.D. \\
\hline & & & {$[\mathrm{M}+\mathrm{Na}]^{+}$} & 310.044784 & N.D. & N.D. & N.D. & N.D. \\
\hline & delphinidin & $\mathrm{C}_{15} \mathrm{H}_{11} \mathrm{O}_{7}$ & $\mathrm{M}^{+}$ & 303.049929 & ++++ & N.D. & +++ & N.D. \\
\hline & & & {$[\mathrm{M}+\mathrm{Na}]^{+}$} & 326.039698 & N.D. & N.D. & N.D. & N.D. \\
\hline & malvidin & $\mathrm{C}_{17} \mathrm{H}_{15} \mathrm{O}_{7}$ & $\mathrm{M}^{+}$ & 331.081229 & +++ & N.D. & t+t+ & ++ \\
\hline & & & {$[\mathrm{M}+\mathrm{Na}\rceil^{+}$} & 354.070999 & N.D. & N.D. & N.D. & N.D. \\
\hline \multirow{6}{*}{ anthocyanin } & cyanidin 3,5 -di- $O$-glucoside & $\mathrm{C}_{27} \mathrm{H}_{31} \mathrm{O}_{16}$ & $\mathrm{M}^{+}$ & 611.160661 & ++++ & + & ++++ & ++ \\
\hline & & & {$[\mathrm{M}+\mathrm{Na}]^{+}$} & 634.150431 & ++++ & N.D. & +++ & ++ \\
\hline & malvidin 3,5-O $O$-diglucoside & $\mathrm{C}_{29} \mathrm{H}_{35} \mathrm{O}_{17}$ & $\mathrm{M}^{+}$ & 655.186876 & ++++ & + & +++ & ++ \\
\hline & & & {$[\mathrm{M}+\mathrm{Na}]^{+}$} & 678.176645 & +++ & N.D. & ++++ & N.D. \\
\hline & peonidin 3,5-O-di-glucopyranoside & $\mathrm{C}_{28} \mathrm{H}_{33} \mathrm{O}_{16}$ & $\mathrm{M}^{+}$ & 625.176311 & +++ & + & t+t+ & ++ \\
\hline & & & {$[\mathrm{M}+\mathrm{Na}]^{+}$} & 648.166081 & N.D. & N.D. & ++++ & N.D. \\
\hline \multirow{6}{*}{ flavonoid aglycone } & apigenin & $\mathrm{C}_{15} \mathrm{H}_{10} \mathrm{O}_{5}$ & {$[\mathrm{M}+\mathrm{H}]^{+}$} & 271.060100 & ++++ & + & +++ & ++ \\
\hline & & & {$[\mathrm{M}+\mathrm{Na}]^{+}$} & 293.042044 & ++++ & N.D. & +++ & ++ \\
\hline & kaempferol & $\mathrm{C}_{15} \mathrm{H}_{10} \mathrm{O}_{6}$ & {$[\mathrm{M}+\mathrm{H}]^{+}$} & 287.055014 & +++ & N.D. & +r++ & ++ \\
\hline & & & {$[\mathrm{M}+\mathrm{Na}]^{+}$} & 309.036959 & ++++ & N.D. & +++ & ++ \\
\hline & quercetin & $\mathrm{C}_{15} \mathrm{H}_{10} \mathrm{O}_{7}$ & {$[\mathrm{M}+\mathrm{H}]^{+}$} & 303.049929 & ++++ & + & +++ & ++ \\
\hline & & & {$[\mathrm{M}+\mathrm{Na}]^{+}$} & 325.031873 & ++++ & N.D. & +++ & + \\
\hline \multirow{6}{*}{ flavonoid glycoside } & homoorientin & $\mathrm{C}_{21} \mathrm{H}_{20} \mathrm{O}_{11}$ & $\lceil\mathrm{M}+\mathrm{H}\rceil^{+}$ & 449.107838 & ++++ & + & +++ & ++ \\
\hline & & & {$[\mathrm{M}+\mathrm{Na}]^{+}$} & 471.089782 & ++++ & + & +++ & ++ \\
\hline & kaempferol 3-O-glucoside & $\mathrm{C}_{21} \mathrm{H}_{20} \mathrm{O}_{11}$ & {$[\mathrm{M}+\mathrm{H}]^{+}$} & 449.107838 & +++ & N.D. & ++ & ++++ \\
\hline & & & $\lceil\mathrm{M}+\mathrm{Na}\rceil^{+}$ & 471.089782 & $++1+$ & + & +r+ & + \\
\hline & rutin & $\mathrm{C}_{27} \mathrm{H}_{30} \mathrm{O}_{16}$ & {$[\mathrm{M}+\mathrm{H}]^{+}$} & 611.160661 & ++ & + & +++ & ++++ \\
\hline & & & {$[\mathrm{M}+\mathrm{Na}]^{+}$} & 633.142606 & ++ & + & ++++ & +++ \\
\hline \multirow{6}{*}{ glucosinolate } & butenyl glucosinolate & $\mathrm{C}_{11} \mathrm{H}_{19} \mathrm{NO}_{9} \mathrm{~S}_{3}$ & $\lceil\mathrm{M}+\mathrm{H}\rceil^{+}$ & 406.029471 & N.D. & N.D. & N.D. & N.D. \\
\hline & & & {$[\mathrm{M}+\mathrm{Na}]^{+}$} & 428.011415 & N.D. & N.D. & N.D. & N.D. \\
\hline & 4-methylthiobutyl glucosinolate & $\mathrm{C}_{12} \mathrm{H}_{23} \mathrm{NO}_{9} \mathrm{~S}_{3}$ & {$[\mathrm{M}+\mathrm{H}]^{+}$} & 422.060771 & N.D. & N.D. & N.D. & N.D. \\
\hline & & & $\lceil\mathrm{M}+\mathrm{Na}]^{+}$ & 444.042715 & N.D. & N.D. & N.D. & N.D. \\
\hline & sinigrin & $\mathrm{C}_{10} \mathrm{H}_{17} \mathrm{NO}_{9} \mathrm{~S}_{2}$ & {$[\mathrm{M}+\mathrm{H}]^{+}$} & 360.041750 & N.D. & N.D. & N.D. & N.D. \\
\hline & & & {$[\mathrm{M}+\mathrm{Na}]^{+}$} & 382.023694 & N.D. & N.D. & N.D. & N.D. \\
\hline \multirow{6}{*}{ lignan } & lariciresinol & $\mathrm{C}_{20} \mathrm{H}_{24} \mathrm{O}_{6}$ & {$[\mathrm{M}+\mathrm{H}]^{+}$} & 361.164565 & N.D. & N.D. & N.D. & N.D. \\
\hline & & & {$[\mathrm{M}+\mathrm{Na}]^{+}$} & 383.146509 & ++++ & N.D. & +++ & ++ \\
\hline & pinoresinol & $\mathrm{C}_{20} \mathrm{H}_{22} \mathrm{O}_{6}$ & {$[\mathrm{M}+\mathrm{H}]^{+}$} & 359.148915 & +++ & N.D. & ++++ & N.D. \\
\hline & & & {$[\mathrm{M}+\mathrm{Na}]^{+}$} & 381.130859 & +++ & N.D. & ++++ & N.D. \\
\hline & secoisolariciresinol diglucoside & $\mathrm{C}_{32} \mathrm{H}_{46} \mathrm{O}_{16}$ & {$[\mathrm{M}+\mathrm{H}]^{+}$} & 687.285862 & N.D. & N.D. & N.D. & N.D. \\
\hline & & & {$[\mathrm{M}+\mathrm{Na}]^{+}$} & 709.267806 & ++ & + & ++++ & +++ \\
\hline \multirow{6}{*}{ phenolamide/hydroxycinnamic acid amide } & $N$-p-coumaroyltyramine & $\mathrm{C}_{17} \mathrm{H}_{17} \mathrm{NO}_{3}$ & {$[\mathrm{M}+\mathrm{H}]^{+}$} & 284.128120 & ++++ & N.D. & ++++ & ++ \\
\hline & & & {$[\mathrm{M}+\mathrm{Na}]^{+}$} & 306.110064 & +++ & N.D. & ++++ & ++ \\
\hline & kukoamin A & $\mathrm{C}_{28} \mathrm{H}_{42} \mathrm{~N}_{4} \mathrm{O}_{6}$ & {$[\mathrm{M}+\mathrm{H}]^{+}$} & 531.317712 & ++ & + & +++t & +++ \\
\hline & & & {$[\mathrm{M}+\mathrm{Na}]^{+}$} & 553.299656 & ++ & N.D. & t+rt & +++ \\
\hline & tricoumaroyl spermidine & $\mathrm{C}_{34} \mathrm{H}_{37} \mathrm{~N}_{3} \mathrm{O}_{6}$ & {$[\mathrm{M}+\mathrm{H}]^{+}$} & 584.275512 & +++ & + & ++++ & ++ \\
\hline & & & {$[\mathrm{M}+\mathrm{Na}]^{+}$} & 606.257457 & +++ & + & ++++ & ++ \\
\hline & chlorogenic acid & $\mathrm{C}_{16} \mathrm{H}_{18} \mathrm{O}_{9}$ & {$[\mathrm{M}+\mathrm{H}]^{+}$} & 355.102359 & N.D. & N.D. & $+++1+$ & N.D. \\
\hline & & & $\lceil\mathrm{M}+\mathrm{Na}\rceil^{+}$ & 377.084303 & +++ & N.D. & N.D. & ++++ \\
\hline phenylnronanoid/coumarin & imperatorin & $\mathrm{C}_{16} \mathrm{H}_{14} \mathrm{O}_{4}$ & {$[\mathrm{M}+\mathrm{H}]^{+}$} & 271.096485 & ++++ & N.D. & +++ & ++ \\
\hline phenylpropanoid/coumarın & & & {$[\mathrm{M}+\mathrm{Na}]^{+}$} & 293.078430 & +++ & N.D. & H+t+ & ++ \\
\hline & sinapic acid & $\mathrm{C}_{11} \mathrm{H}_{12} \mathrm{O}_{5}$ & $\lceil\mathrm{M}+\mathrm{H}\rceil^{+}$ & 225.075750 & ++++ & +++ & N.D. & N.D. \\
\hline & & & {$[\mathrm{M}+\mathrm{Na}]^{+}$} & 247.057694 & N.D. & ++++ & N.D. & N.D. \\
\hline & sarsasapogenin & $\mathrm{C}_{27} \mathrm{H}_{44} \mathrm{O}_{3}$ & {$[\mathrm{M}+\mathrm{H}]^{+}$} & 417.336322 & ++ & N.D. & ++++ & +++ \\
\hline & & & {$[\mathrm{M}+\mathrm{Na}]^{+}$} & 439.318266 & N.D. & N.D. & ++++ & N.D. \\
\hline sanonin/olycoalkaloid aglycone & soyasapogenol A & $\mathrm{C}_{30} \mathrm{H}_{50} \mathrm{O}_{4}$ & {$[\mathrm{M}+\mathrm{H}]^{+}$} & 475.378187 & N.D. & N.D. & N.D. & N.D. \\
\hline saponın/glycoalkaloid aglycone & & & {$[\mathrm{M}+\mathrm{Na}]^{+}$} & 497.360131 & +++ & N.D. & ++++ & ++ \\
\hline & tomatidine & $\mathrm{C}_{27} \mathrm{H}_{45} \mathrm{NO}_{2}$ & {$[\mathrm{M}+\mathrm{H}]^{+}$} & 416.352306 & +++ & ++ & ++++ & N.D. \\
\hline & & & {$[\mathrm{M}+\mathrm{Na}]^{+}$} & 438.334250 & ++ & + & +++ & ++++ \\
\hline & ginsenoside Ro & $\mathrm{C}_{48} \mathrm{H}_{76} \mathrm{O}_{19}$ & {$[\mathrm{M}+\mathrm{H}]^{+}$} & 957.505357 & N.D. & N.D. & N.D. & N.D. \\
\hline & & & {$[\mathrm{M}+\mathrm{Na}]^{+}$} & 979.487301 & +++ & ++ & ++++ & + \\
\hline sanonin/alycoalkaloid & soyasaponin $\mathrm{Bb}$ & $\mathrm{C}_{48} \mathrm{H}_{78} \mathrm{O}_{18}$ & {$[\mathrm{M}+\mathrm{H}]^{+}$} & 943.526092 & + & ++ & +++ & ++++ \\
\hline saponin/glycoalkaloid & & & {$[\mathrm{M}+\mathrm{Na}]^{+}$} & 965.508036 & ++ & + & ++++ & +++ \\
\hline & tomatine & $\mathrm{C}_{50} \mathrm{H}_{83} \mathrm{NO}_{21}$ & {$[\mathrm{M}+\mathrm{H}]^{+}$} & 1034.553035 & +++ & + & ++++ & ++ \\
\hline & & & {$[\mathrm{M}+\mathrm{Na}]^{+}$} & 1056.534979 & +++ & + & ++ & ++++ \\
\hline & g-glutamylcysteine & $\mathrm{C}_{8} \mathrm{H}_{14} \mathrm{~N}_{2} \mathrm{O}_{5} \mathrm{~S}$ & {$[\mathrm{M}+\mathrm{H}]^{+}$} & 251.069619 & N.D. & N.D. & ++++ & N.D. \\
\hline & & & {$[\mathrm{M}+\mathrm{Na}]^{+}$} & 273.051563 & N.D. & N.D. & N.D. & N.D. \\
\hline sulfur-containing metabolite & glutathione & $\mathrm{C}_{10} \mathrm{H}_{17} \mathrm{~N}_{3} \mathrm{O}_{6} \mathrm{~S}$ & {$[\mathrm{M}+\mathrm{H}]^{+}$} & 308.091083 & ++ & N.D. & ++++ & +++ \\
\hline sulfur-containing metabolite & & & {$[\mathrm{M}+\mathrm{Na}]^{+}$} & 330.073027 & +++ & N.D. & ++++ & ++ \\
\hline & 1-isothiocyanato-9-(methylsulfinyl)- & $\mathrm{C}_{11} \mathrm{H}_{21} \mathrm{NOS}_{2}$ & {$[\mathrm{M}+\mathrm{H}]^{+}$} & 248.113733 & ++++ & N.D. & +r+ & ++ \\
\hline & & & {$[\mathrm{M}+\mathrm{Na}]^{+}$} & 270.095677 & ++ & N.D. & ++++ & +++ \\
\hline
\end{tabular}

N.D., not detected.

,,++++++ , and +++ can be compared among the matrix reagents, not other metabolites. 
stress. Plants typically accumulate such metabolites within their outermost layers (e.g., epidermis). Spatial data will be an important key for understanding the function of metabolites. The role of specialized metabolites remains unknown within many tissues. Hopefully, the active participation of IMS analysis in phytochemical genomics will help determine their roles.

\section{ACKNOWLEDGEMENTS}

This work was partially supported by JST the Strategic International Collaborative Research Program, MAFF Science and Technology Research Promotion Program for Agriculture, Forestry, Fisheries and Food Industry, and the Japan Advanced Plant Science Network.

\section{REFERENCES}

Addie, R. D., Balluff, B., Bovee, J. V., Morreau, H., and McDonnell, L. A. (2015) Current state and future challenges of mass spectrometry imaging for clinical research. Anal Chem 87: 6426-6433.

Alseekh, S., and Fernie, A. R. (2018) Metabolomics 20 years on: what have we learned and what hurdles remain? Plant $J$ 94: 933-942.

Baker, T. C., Han, J., and Borchers, C. H. (2017) Recent advancements in matrix-assisted laser desorption/ionization mass spectrometry imaging. Curr Opin Biotechnol 43: 6269.

Boughton, B. A., Thinagaran, D., Sarabia, D., Bacic, A., and Roessner, U. (2016) Mass spectrometry imaging for plant biology: a review. Phytochem Rev 15: 445-488.

Buchberger, A. R., DeLaney, K., Johnson, J., and Li, L. (2018) Mass spectrometry imaging: A review of emerging advancements and future insights. Anal Chem 90: 240-265.

Colinas, M., and Goossens, A. (2018) Combinatorial transcriptional control of plant specialized metabolism. Trends Plant Sci 23: 324-336.

Dong, Y., Li, B., Malitsky, S., Rogachev, I., Aharoni, A., Kaftan, F., Svatos, A., and Franceschi, P. (2016) Sample preparation for mass spectrometry imaging of plant tissues: A review. Front Plant Sci 7: 60.

Duenas, M. E., Klein, A. T., Alexander, L. E., Yandeau-Nelson, M. D., Nikolau, B. J., and Lee, Y. J. (2017) High spatial resolution mass spectrometry imaging reveals the genetically programmed, developmental modification of the distribution of thylakoid membrane lipids among individual cells of maize leaf. Plant J 89: 825-838.

Etalo, D. W., De Vos, R. C. H., Joosten, M. H. A. J., and Hall, R. D. (2015) Spatially resolved plant metabolomics: Some potentials and limitations of laser-ablation electrospray ionization mass spectrometry metabolite imaging. Plant Physiol 169: 1424-1435.

Fernie, A. R., and Tohge, T. (2017) The genetics of plant metabolism. Annu Rev Genet 51: 287-310.

Freund, D. M., and Hegeman, A. D. (2017) Recent advances in stable isotope-enabled mass spectrometry-based plant metabolomics. Curr Opin Biotechnol 43: 41-48.
Fujimura, Y., and Miura, D. (2014) MALDI mass spectrometry imaging for visualizing in situ metabolism of endogenous metabolites and dietary phytochemicals. Metabolites 4: 319346.

Giavalisco, P., Li, Y., Matthes, A., Eckhardt, A., Hubberten, H. M., Hesse, H., Segu, S., Hummel, J., Kohl, K., and Willmitzer, L. (2011) Elemental formula annotation of polar and lipophilic metabolites using C-13, N-15 and S-34 isotope labelling, in combination with high- resolution mass spectrometry. Plant $J$ 68: 364-376.

Glaser, K., Kanawati, B., Kubo, T., Schmitt-Kopplin, P., and Grill, E. (2014) Exploring the Arabidopsis sulfur metabolome. Plant J 77: 31-45.

Hautbergue, T., Jamin, E. L., Costantino, R., Tadrist, S., Meneghetti, L., Tabet, J. C., Debrauwer, L., Oswald, I. P., and Puel, O. (2019) Combination of isotope labeling and molecular networking of tandem mass spectrometry data to reveal 69 unknown metabolites produced by Penicillium nordicum. Anal Chem 91: 12191-12202.

Huang, A. C., Jiang, T., Liu, Y. X., Bai, Y. C., Reed, J., Qu, B., Goossens, A., Nutzmann, H. W., Bai, Y., and Osbourn, A. (2019) A specialized metabolic network selectively modulates Arabidopsis root microbiota. Science 364.

Kera, K., Fine, D. D., Wherritt, D. J., Nagashima, Y., Shimada, N., Ara, T., Ogata, Y., Sumner, L. W., and Suzuki, H. (2018) Pathway-specific metabolome analysis with ${ }^{18} \mathrm{O}_{2}$-labeled Medicago truncatula via a mass spectrometry-based approach. Metabolomics 14: 71.

Kompauer, M., Heiles, S., and Spengler, B. (2017) Atmospheric pressure MALDI mass spectrometry imaging of tissues and cells at 1.4- $\mu \mathrm{m}$ lateral resolution. Nat Methods 14: 90-96.

Kompauer, M., Heiles, S., and Spengler, B. (2017) Autofocusing MALDI mass spectrometry imaging of tissue sections and 3D chemical topography of nonflat surfaces. Nat Methods 14: 1156-1158.

Lee, Y. J., Perdian, D. C., Song, Z. H., Yeung, E. S., and Nikolau, B. J. (2012) Use of mass spectrometry for imaging metabolites in plants. Plant $J$ 70: 81-95.

Lei, Z., Huhman, D. V., and Sumner, L. W. (2011) Mass spectrometry strategies in metabolomics. $J$ Biol Chem 286: 25435-25442.

Li, B., Bhandari, D. R., Janfelt, C., Rompp, A., and Spengler, B. (2014) Natural products in Glycyrrhiza glabra (licorice) rhizome imaged at the cellular level by atmospheric pressure matrix-assisted laser desorption/ionization tandem mass spectrometry imaging. Plant $J$ 80: 161-171.

Li, D. P., Heiling, S., Baldwin, I. T., and Gaquerel, E. (2016) Illuminating a plant's tissue-specific metabolic diversity using computational metabolomics and information theory. Proc Natl Acad Sci USA 113: E7610-E7618.

Massalha, H., Korenblum, E., Tholl, D., and Aharoni, A. (2017) Small molecules below-ground: the role of specialized metabolites in the rhizosphere. Plant J 90: 788-807.

Nakabayashi, R., Hashimoto, K., Toyooka, K., and Saito, K. (2017) Top-down metabolomic approaches for 
nitrogen-containing metabolites. Anal Chem 89: 2698-2703.

Nakabayashi, R., Hashimoto, K., Toyooka, K., and Saito, K. (2019) Keeping the shape of plant tissue for visualizing metabolite features in segmentation and correlation analysis of imaging mass spectrometry in Asparagus officinalis. Metabolomics 15: 24.

Nakabayashi, R., and Saito, K. (2015) Integrated metabolomics for abiotic stress responses in plants. Curr Opin Plant Biol 24: 10-16.

Nakabayashi, R., Sawada, Y., Yamada, Y., Suzuki, M., Hirai, M. Y., Sakurai, T., and Saito, K. (2013) Combination of liquid chromatography-Fourier transform ion cyclotron resonance-mass spectrometry with ${ }^{13} \mathrm{C}$-labeling for chemical assignment of sulfur-containing metabolites in onion bulbs. Anal Chem 85: 1310-1315.

Nakabayashi, R., Tsugawa, H., Mori, T., and Saito, K. (2016) Automation of chemical assignment for identifying molecular formula of S-containing metabolites by combining metabolomics and chemoinformatics with ${ }^{34} \mathrm{~S}$ labeling. Metabolomics 12: 168.

Nakabayashi, R., Yang, Z., Nishizawa, T., Mori, T., and Saito, K. (2015) Top-down targeted metabolomics reveals a sulfur-containing metabolite with inhibitory activity against angiotensin-converting Enzyme in Asparagus officinalis. $J$ Nat Prod 78: 1179-1183.

Nakabayashi, R., Yonekura-Sakakibara, K., Urano, K., Suzuki, M., Yamada, Y., Nishizawa, T., Matsuda, F., Kojima, M., Sakakibara, H., Shinozaki, K., Michael, A. J., Tohge, T., Yamazaki, M., and Saito, K. (2014) Enhancement of oxidative and drought tolerance in Arabidopsis by overaccumulation of antioxidant flavonoids. Plant J 77: 367379.

O’Connor, S. E., and Maresh, J. J. (2006) Chemistry and biology of monoterpene indole alkaloid biosynthesis. Nat Prod Rep 23: 532-547.

Rai, A., Saito, K., and Yamazaki, M. (2017) Integrated omics analysis of specialized metabolism in medicinal plants. Plant $J$ 90: 764-787.

Saito, K. (2013) Phytochemical genomics - a new trend. Curr Opin Plant Biol 16: 373-380.

Saito, K., and Matsuda, F. (2010) Metabolomics for functional genomics, systems biology, and biotechnology. Annu Rev Plant Biol 61: 463-489.

Schroeder, M., Meyer, S. W., Heyman, H. M., Barsch, A., and Sumner, L. W. (2019) Generation of a collision cross section library for multi-dimensional plant metabolomics using UHPLC-trapped ion mobility-MS/MS. Metabolites 10: 13.

Shariatgorji, M., Nilsson, A., Fridjonsdottir, E., Vallianatou, T., Kallback, P., Katan, L., Savmarker, J., Mantas, I., Zhang, X., Bezard, E., Svenningsson, P., Odell, L. R., and Andren, P. E. (2019) Comprehensive mapping of neurotransmitter networks by MALDI-MS imaging. Nat Methods 16: 10211028.

Spraggins, J. M., Djambazova, K. V., Rivera, E. S., Migas, L. G., Neumann, E. K., Fuetterer, A., Suetering, J., Goedecke,
N., Ly, A., Van de Plas, R., and Caprioli, R. M. (2019) High-performance molecular imaging with MALDI trapped ion-mobility time-of-flight (timsTOF) mass spectrometry. Anal Chem 91: 14552-14560.

Suzuki, M., Nakabayashi, R., Ogata, Y., Sakurai, N., Tokimatsu, T., Goto, S., Suzuki, M., Jasinski, M., Martinoia, E., Otagaki, S., Matsumoto, S., Saito, K., and Shiratake, K. (2015) Multiomics in grape berry skin revealed specific induction of the stilbene synthetic pathway by ultraviolet-C irradiation. Plant Physiol 168: 47-59.

Tohge, T., Wendenburg, R., Ishihara, H., Nakabayashi, R., Watanabe, M., Sulpice, R., Hoefgen, R., Takayama, H., Saito, K., Stitt, M., and Fernie, A. R. (2016) Characterization of a recently evolved flavonol-phenylacyltransferase gene provides signatures of natural light selection in Brassicaceae. Nat Commun 7: 12399.

Tsugawa, H. (2018) Advances in computational metabolomics and databases deepen the understanding of metabolisms. Curr Opin Biotechnol 54: 10-17.

Tsugawa, H., Nakabayashi, R., Mori, T., Yamada, Y., Takahashi, M., Rai, A., Sugiyama, R., Yamamoto, H., Nakaya, T., Yamazaki, M., Kooke, R., Bac-Molenaar, J. A., OztolanErol, N., Keurentjes, J. J. B., Arita, M., and Saito, K. (2019) A cheminformatics approach to characterize metabolomes in stable-isotope-labeled organisms. Nat Methods 16: 295-298.

Wang, Z. Z., and Jones, A. D. (2014) Profiling of Stable Isotope Enrichment in Specialized Metabolites Using Liquid Chromatography and Multiplexed Nonselective CollisionInduced Dissociation. Anal Chem 86: 10600-10607.

Wolfender, J. L., Nuzillard, J. M., van der Hooft, J. J. J., Renault, J. H., and Bertrand, S. (2019) Accelerating Metabolite Identification in Natural Product Research: Toward an Ideal Combination of Liquid Chromatography-High-Resolution Tandem Mass Spectrometry and NMR Profiling, in Silico Databases, and Chemometrics. Anal Chem 91: 704-742.

Yamamoto, K., Takahashi, K., Caputi, L., Mizuno, H., Rodriguez-Lopez, C. E., Iwasaki, T., Ishizaki, K., Fukaki, H., Ohnishi, M., Yamazaki, M., Masujima, T., O'Connor, S. E., and Mimura, T. (2019) The complexity of intercellular localisation of alkaloids revealed by single-cell metabolomics. New Phytol 224: 848-859.

Yamamoto, K., Takahashi, K., Mizuno, H., Anegawa, A., Ishizaki, K., Fukaki, H., Ohnishi, M., Yamazaki, M., Masujima, T., and Mimura, T. (2016) Cell-specific localization of alkaloids in Catharanthus roseus stem tissue measured with Imaging MS and Single-cell MS. Proc Natl Acad Sci USA 113: 3891-3896.

Yonekura-Sakakibara, K., Tohge, T., Matsuda, F., Nakabayashi, R., Takayama, H., Niida, R., Watanabe-Takahashi, A., Inoue, E., and Saito, K. (2008) Comprehensive flavonol profiling and transcriptome coexpression analysis leading to decoding gene-metabolite correlations in Arabidopsis. Plant Cell 20: 2160-2176

Received: 24 January 2020 / Accepted: 11 March 2020 